
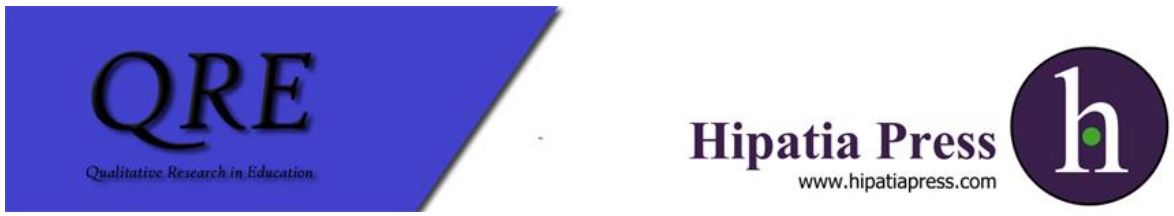

Instructions for authors, subscriptions and further details:

http://qre.hipatiapress.com

\title{
Formarse en la Investigación Educativa: una Comunidad de Pensamiento en Torno a la Escritura de la Tesis Doctoral
}

Emma Quiles-Fernández ${ }^{1}$, Julio Hizmeri Fernández ${ }^{2}$ \& Roxana Hormazábal Fajardo ${ }^{3}$

1) Centre for Research for Teacher Education and Development, University of Alberta, Canada.

2) Departmento de Didáctica, Universidad Católica de la Santísima Concepción, Chile.

3) Facultad de Pedagogía, Universidad Academia de Humanismo Cristiano, Chile.

Date of publication: October $28^{\text {th }}, 2018$

Edition period: October 2018 - February 2019

To cite this article: Quiles-Fernández, E., Hizmeri, J., \& Hormazábal Fajardo, R. (2018). Formarse en la investigación educativa: una comunidad de pensamiento en torno a la escritura de la tesis doctoral. Qualitative Research in Education, 7(3), 241-264. doi:10.17583/qre.2018.3482

To link this article: http://dx.doi.org/10.17583/qre.2018.3482

\section{PLEASE SCROLL DOWN FOR ARTICLE}

The terms and conditions of use are related to the Open Journal System and to Creative Commons Attribution License (CC-BY). 


\section{Learning about Educational Research: a Community of} Knowledge towards the Writing Developed during our Doctoral Work

Emma Quiles-Fernández University of Alberta
Julio Hizmeri Fernández

Universidad Católica de la Santísima Concepción

Roxana Hormazábal Fajardo

Universidad Academia de

Humanismo Cristiano

(Received: 02 May 2018; Accepted: 18 October 2018; Published: 28

October 2018)

\section{Abstract}

This article explores educative experiences we had as doctoral students in a community of knowledge inside the University of Barcelona. We deepen understandings around the process we lived for five years, as well as we point out theoretical and methodological aspects that framed the process itself. Embracing narrative inquiry as methodology, we enhance the need of shifting some of the doctoral training practices that traditional academic systems still hold. Through our stories, we show bumps and tensions that might emerge in living and working in a community of knowledge. We also raise challenges that beginning researchers are currently facing in the educational landscape. Those challenges are related to the ways in which we inquire, approach, attend to, and name the research experience in the context of increasingly high academic demands.

Keywords: higher education, doctoral degree, educational practice, narrative inquiry 


\section{Formarse en la Investigación Educativa: una Comunidad de Pensamiento en Torno a la Escritura de la Tesis Doctoral}

Emma Quiles-Fernández

University of Alberta

Roxana Hormazábal Fajardo

Universidad Academia de

Humanismo Cristiano

\author{
Julio Hizmeri Fernández \\ Universidad Católica de la Santísima \\ Concepción
}

(Recibido: 02 de mayo de 2018; Aceptado: 18 de octubre de 2018; Publicado: 28 de octubre de 2018)

\section{Resumen}

El artículo recoge nuestra experiencia formativa como estudiantes de doctorado en una comunidad de pensamiento creada en la Universidad de Barcelona. En él exploramos y narramos el proceso que vivimos durante cinco años en dicha comunidad, así como damos cuenta de las claves teóricas y metodológicas que orientaron nuestro trabajo. Sostenidos en la metodología de indagación narrativa, realzamos la necesidad de transformar algunas de las prácticas de formación doctoral que continúan bajo un modelo académico más tradicional. En una narrativa colectiva que, en momentos, también es planteada desde nuestras voces individuales, abordamos posibles limitaciones, alcances y tensiones que pueden emerger en este tipo de experiencias de formación, al tiempo que planteamos algunos desafíos a los que se enfrentan quienes se inician en la investigación educativa, especialmente en lo que concierne a los modos de indagar (mirar, pensar, nombrar lo educativo) en un contexto signado por las altas exigencias académicas vinculadas a la escritura de una tesis doctoral.

Palabras clave: enseñanza superior, doctorado, práctica educativa, indagación narrativa

2018 Hipatia Press

ISSN: 2014-6418

DOI: 10.17583 /qre.2018.3482 


\section{ntonia De Vita (2012), en su libro La creación social. Relaciones y contextos para educar, señala que:}

más allá de la línea recta para alcanzar un resultado (...) hay una educación en el proceso, un aprender en el transcurso del camino, que llega antes que la meta. Aprender al recorrer un camino tiene que ver con encontrar el propio lugar, con un situarse, con la búsqueda de una relación propia con las cosas que hacemos (p.108).

Esta noción de proceso, crecimiento y búsqueda continua de un lugar propio es lo que nos empujó a crear Huerting, una comunidad de pensamiento en la que poder compartir experiencias de lectura, saber y escritura que iban emergiendo del trabajo de nuestras tesis doctorales. Las palabras de De Vita nos posibilitan nombrar ese espacio educativo en el que fuimos compartiendo no solo anhelos e historias, sino la necesidad de sentirnos parte de una comunidad que iba más allá de nuestras investigaciones individuales y del mundo académico, así como repensar la relación que teníamos con nuestros propósitos, con lo que deseábamos preguntarnos y con el viaje investigativo que llevábamos a cabo junto a maestros y maestras. Sobre todo, fu un lugar para repensarnos, en un camino en el que continuamente nos íbamos haciendo, componiendo, llegando a ser (Greene, 1995).

En este artículo deseamos explorar y dar cuenta de los procesos de la comunidad de pensamiento que creamos y en la que participamos durante cinco años, así como el sentido educativo e investigativo que dicho espacio ha supuesto en nuestro desarrollo profesional. Revisitar la experiencia y la escritura elaborada, fruto de los encuentros en Huerting, nos ha permitido atender a nuestras actuales responsabilidades académicas desde la necesidad de repensar la formación de "investigadoras nacientes" (Hormazábal Fajardo, 2016) ${ }^{1}$, pudiendo incorporar y crear otros espacios de crecimiento profesional desde los que sabemos que es posible habitar la academia. 


\section{Situando nuestra Comunidad de Pensamiento}

Revisitamos y escribimos acerca de nuestra comunidad de pensamiento, ahora desde la distancia temporal y como formadores en diferentes contextos universitarios e investigadores ya graduados, porque entendemos que aún resuena en cada uno de nosotros el sentido formativo de Huerting. Un sentido que compartimos en la academia y que fue propiciada por la indagación narrativa de la experiencia ${ }^{2}$. Una experiencia que, por cierto, aún continúa invitándonos a pensar en el sentido y el significado de la narrativa en la construcción de saberes para el ámbito educativo tanto desde lo epistemológico como lo metodológico.

Iniciamos este recorrido en 2011 con la invitación de José Contreras, director de nuestras tesis doctorales. Bajo la idea de "favorecer un espacio de relación e intercambio frente al sentimiento de soledad que suele acompañar el proceso de realización de la tesis" (Contreras, 2014, p.3), siete estudiantes de doctorado nos reunimos mensualmente con el deseo de continuar despertando esa chispa que incita a la curiosidad, a la lectura, a un nuevo punto de vista y a la interrogación que aviva el deseo de saber. Empezamos los encuentros junto a Amparo Chumacero, Patricia Gabbarini, Roxana Hormazábal Fajardo, Emma Quiles-Fernández y Gonzalo Urra, a los que más tarde se añadieron Juliana Machado y Julio Hizmeri Fernández. La idea era que "el propio grupo fuera marcando su camino" (Contreras, 2014, p.3), es decir, que el acompañamiento del director se caracterizase por estar junto a nosotros, aprendiendo y preguntándose del mismo modo en que nosotros lo hacíamos en nuestro proceso formativo.

En este sentido, la comunidad de pensamiento se caracterizó por un estar-en-común que no estuvo determinado desde una esencia, ni como un programa predefinido, más que el simple estar juntos (Nancy, 2000), en una asociación libre en torno a la escritura de la tesis entre quienes comparten el deseo de habitar la universidad de manera creativa con modalidades menos académicas:

una pequeña comunidad de auto-mutua-ayuda que con sencillez te saca del aislamiento, de la vivencia de que las dificultades de las tesis solo las tienes tú, y que te permite, por la puesta en común de los problemas y de las cosas que van bien, poder recoger los frutos de quienes van más avanzados que tú, creando un círculo virtuoso 
de ideas y de pensamientos para las tesis en un lugar social de relaciones, confrontación e intercambios (De Vita, 2002, p. 112).

Favorecer dicho espacio relacional permitía la creación de un lugar de autoformación y formación nutrido por un intercambio vivo desde el que cada aporte alentaba una búsqueda de sentido que conjugaba procesos singulares de investigación con nuevas aperturas nacidas del estar singularplural (Nancy, 2000). Fruto de nuestras investigaciones de doctorado, historias de vida y experiencias educativas, el estar juntos nos permitió "pensar en presencia" (Zamboni, 2009) y "saber en relación" (Arnaus, 2013), dando lugar a un espacio simbólico en el que nuestras experiencias se hacían presentes y nos dejábamos decir por ellas.

Así, a través de un pensamiento relacional que nos ofrecía una salida a la práctica de escritura, generalmente solitaria, en la academia, cada encuentro nos llevaba por derroteros indeterminados en los que nos íbamos conociendo y reconociendo y en los que fuimos trazando un camino formativo $^{3}$ en el que, lentamente, nos íbamos haciendo conscientes de aquello a lo que cada uno trataba de ir dando sentido y significado en su investigación doctoral.

En 2012 surgió la necesidad de darnos un nombre como grupo porque cuando alguien externo preguntaba quiénes éramos, solíamos responder con expresiones como "grupo de seminario" o "grupo de trabajo" sin estar muy convencidos de aquello; se hacía necesario un nombre que expresara el trabajo que veníamos haciendo. Es ahí cuando, mirando el etiquetado de un refresco, Gonzalo Urra propuso el nombre de Huerting ${ }^{4}$. De manera unánime nos apropiamos de él, percibiendo que llevaba consigo el gesto de labrar, regar y dar tiempo a la siembra. Un gesto que dice mucho de lo que hemos tratado de entretejer como proceso formativo comunitario. Sentíamos que lo que nos unía era el cultivo por nuestros trabajos y por el saber educativo, y gracias a él cuidábamos la relación. Una relación que nos ha transformado (y lo continúa haciendo). En Huerting había un deseo de comprendernos y acompañarnos, de estar ahí y cultivar el acto de pensar en presencia (Zamboni, 2009). Esto se daba a través del silencio, de poner en juego palabras de apertura y de la escucha; y, finalmente, con la vivencia de la voz como aquello que nos mostraba algo del camino que nos hacía avanzar en nuestras investigaciones. 
Debido a experiencias universitarias pasadas, sabíamos que las formas institucionales, a menudo, limitan y dificultan los procesos de investigación, imponiendo ritmos y prácticas que desatienden las necesidades reales de los proyectos personales. Ahora, al retomar nuestros escritos, tomamos conciencia de que la experiencia de Huerting se ha dado en un margen visible, fértil, autónomo que, si bien ha estado alejado de algunas lógicas academicistas, su creación ha posibilitado la creación de un saber pedagógico, de la experiencia y, por tanto, académico. En este sentido, la periferia habitada se convirtió en un centro significativo de encuentro y crecimiento profesional y personal, y es que, "según se mire, uno siempre está ocupando múltiples centros y periferias superpuestos simultáneamente" (Porres, 2012, p.39). Así, lo que ha mantenido viva la comunidad ha sido la necesidad de tener un espacio al que siempre regresar cuando la soledad de la escritura o las presiones académicas se hacían presentes.

Ocupar el lugar desde esa periferia fructífera, permitía hacer un desplazamiento de lo instituido para cuidar y atender a nuestros propios ritmos, posibilitando un modo diverso de estar y de relacionarnos con nuevas prácticas de saber. Y abrazar ese camino ha posibilitado que la labor investigativa fuera acogida como una experiencia en sí, problematizando continuamente los supuestos teóricos, metodológicos, relacionales que emergen durante el trabajo investigativo. Una experiencia, por tanto, en la que también hemos sentido vulnerabilidad porque, a pesar de sostenernos en un determinado horizonte teórico, el acto de dejarnos tocar por los otros, así como de sentirnos perdidos en el camino para descubrir otros modos de indagar, nos ha permitido vivir un movimiento y desorientación necesarios para nuestro quehacer investigativo (Behar, 1996; Rubinstein-Avila \& Maranzana, 2015). Mas, en este andar, la experiencia no ha prescindido de un método o de un horizonte teórico que ha guiado el camino, pues como señala María Zambrano (2011):

la verdadera experiencia no puede darse sin la intervención de un tipo de método. El método ha debido estar desde un principio en una cierta y determinada experiencia, que por la virtud de aquél llega a cobrar cuerpo y forma, figura (p.68). 
Así, en nuestro proceso de búsqueda ha sido necesario acompañarnos de un conjunto de autoras y autores y sus pensamientos que, en forma de señales teóricas, nos proporcionaron una determinada experiencia de camino, un modo particular para recorrerlo y una atenta mirada a la educación. Todo ello ha creado un paisaje de cuatro orientaciones teóricas que han venido guiando nuestras preguntas acerca de la formación docente y la indagación educativa.

a) Aprender más allá de la razón tradicional. Sabemos que la formación académica se ha ubicado en lo que podría denominarse el espacio de la razón tradicional, que ha violentado a la experiencia, mostrándose desapegada de la vida (Zambrano, 2004). Y el pensamiento calculador de la ciencia moderna (Heidegger, 1978), que desconfía de la experiencia, ha necesitado, para conocer y apropiarse del mundo, la separación del sujeto que piensa con la cosa pensada, dejando a quien piensa fuera de lo pensado, y al pensamiento despoblado de su subjetividad, renunciando al hecho de poder habitarlas y dotarlas de sentido (Merleau-Ponty, 2013).

Este proceso de desgarramiento o separación lo hemos experimentado desde nuestras trayectorias escolares, donde aprendimos a conocer la realidad desde una impersonal distancia. No obstante, hoy, una educación y una investigación de y desde la experiencia (Huber, Caine, Huber, \& Steeves, 2014), entendiendo por experiencia "eso que nos pasa; no lo que pasa, sino eso que me pasa" (Larrosa, 2003, p.166), nos ha permitido pensar en lo que nos pasa y dotar de sentido a lo vivido, partiendo de sí (Piussi, 2000) para llevarlo a esos espacios académicos desde nuestra actual labor como formadoras de formadores, intentando generar otros modos de aprender más allá de lo tradicional.

b) Aprender que no podemos desligarnos de lo vivido, nos ha permitido pensar la relación entre saber y experiencia (Contreras \& Pérez de Lara, 2010; Contreras, 2013). Mediante las aportaciones y acompañamiento de nuestro director, comprendemos que el saber educativo necesita mantenerse en relación viva con la experiencia, porque desde ella nace la inquietud, la curiosidad y la cuestión pedagógica: la pregunta por el sentido y por lo adecuado. Además, el saber que sostiene el hacer educativo (un hacer concreto, de alguien), nace de lo vivido y de lo pensado como propio (esto es, pensado como una experiencia), y necesita hacer el recorrido de sentido, disposiciones y vivencias que orientan ese hacer personal (Contreras, 2010, p.68). 
Así, el saber educativo tiene lugar a partir de lo que hacemos y lo que nos pasa junto a las niñas, niños, jóvenes, familias y maestros que participan en nuestras investigaciones. Es decir, el saber educativo, en tanto saber de la experiencia, no se encuentra al margen del ser. Aparece en la concreción de una situación singular, fuera de un conocimiento objetivo, generalizable o abstracto, porque se trata de un saber encarnado y corporeizado en primera persona.

c) Y, si lo que hemos intentado es dar cuenta de la experiencia vivida, el modo de nombrar y pensar lo vivido no se ha podido dar fuera del tiempo de la vivencia. Lo vivido, el tiempo humano, se articula en modo narrativo (Ricoeur, 2004; Bárcena \& Mèlich, 2014). Por ello, en Huerting hemos necesitado de la narrativa como forma de dar cuenta de nuestra experiencia investigativa. Entendemos que hay una relación constitutiva entre vivir y narrar y la narración, por lo tanto, a través de nuestras historias investigativas hemos venido tejiendo y ensanchando el sentido educativo de las experiencias vividas.

El motivo principal para el uso de la narrativa en la investigación educativa es que los seres humanos somos organismos contadores de historias que, individual y socialmente, vivimos vidas relatadas. Así, la indagación narrativa se convierte en una oportunidad para estudiar la forma en que los seres humanos experimentamos el mundo (Connelly \& Clandinin, 1995), asumiendo que la razón de ser de cualquier investigación educativa no estriba únicamente en describir o explicar lo que sucede en un espacio educativo, sino en revelar, abrir, suscitar y desvelar la pregunta pedagógica que existe en ella (Clandinin, 1985).

d) Profundizar narrativamente la educación (Contreras \& QuilesFernández, 2017) nos ha ayudado a observar y atender a lo educativo sin fijarlo conceptualmente, intentando rozar su sentido a medida que este se mostraba junto a las narrativas de la vida misma. La profundización narrativa puede ser un modo de sostener un pensar vivo y en movimiento que acompaña al narrar sin interrumpirlo, entrando en él como una voz que reflexiona pedagógicamente al hilo de las historias que se cuentan, buscando luz.

Para nosotros, estas cuatro orientaciones teóricas y experienciales tienen que ver con un giro hacia dar (a) luz a un saber que reconoce aquello que se significa y que queda representado en un cultivo que siempre es naciente y 
que permite observar la realidad educativa como una realidad nueva que aparece ante nosotros.

\section{Nuestro Camino Metodológico}

Revisitando las consideraciones ontológicas y epistemológicas que nos han servido de horizonte teórico, consideramos que el modo en que hemos atendido a la experiencia indagativa abre un doble sentido a lo que nombramos como "investigación educativa" (Simons \& Masschelein, 2006). Por un lado, lo educativo o pedagógico remite a un campo de trabajo, dando lugar en nuestro caso a tres temas o tesis diferentes; por otro lado, lo pedagógico alude a la naturaleza de la investigación en sí, a la dimensión formativa de la actividad misma o a la experiencia de transformación que hemos vivido en el camino.

Entendiendo el método desde su etimología (methodos, camino), la experiencia del camino ha tenido que ver con la experiencia de pasaje o recorrido realizado y con la experiencia de pasión, es decir, de aquello que nos ha ido pasando durante el recorrido mismo. Y donde la escritura, lectura, conversación y reescritura han sido los modos que han signado el camino de Huerting.

El hecho de partir de la escucha y no de la palabra ha resultado una experiencia fundante de especial cuidado y sensibilidad, porque sabíamos de la dificultad que suponía el hecho de dejarse leer. Pues, leer es "hacer de sí un escenario interior (...) un oír interior en el hacerse sonido del lenguaje" (Gadamer, 2000, p.74), o sea, una empresa relacional que nos convocaba a descubrir las voces de quienes escribían y a apropiarnos del sentido que dejan entrever. Leer equivalía a estar atento para capturar el sentido de la realidad; escuchar implicaba dejar que el otro nos interpelara; y comprender nos recordaba la capacidad de relacionar los sentidos de lo que se nos hacía presente.

La composición de Huerting se ha dado en torno a la experiencia de contar, vivir, recontar y revivir relatos de experiencias (Clandinin \& Huber, 2012), entendiendo la narrativa tanto como fenómeno a investigar -la experiencia relatada-, como método -el modo narrativo de profundizar la experiencia-(Connelly \& Clandinin, 1995). Así, en nuestras exploraciones, la indagación narrativa ha tenido que ver con el paisaje como con el modo 
de transitarlo y, aún más, con nuestras propias experiencias formativas a partir de lo que las investigaciones nos fueron mostrando.

En este sentido, la distinción metodológica de este tipo de investigación educativa no radicaría en diferenciar entre investigación cuantitativa versus cualitativa, o empírico-analítica versus interpretativa, sino en relación al lugar y al significado que tiene en cada una de ellas el acceso a la verdad:

según una cierta tradición (...) el acceso a la verdad requiere la transformación del yo (...) la transformación del investigador es imprescindible para poder acceder a la verdad, a un cierto tipo de verdad. La otra tradición, más conocida, plantea que es el conocimiento el que nos permite acceder a la verdad, que hay que cumplir determinadas condiciones para adquirir conocimientos verdaderos, pero que la transformación del investigador no es una de esas condiciones. (Simons \& Masschelein, 2006, p.130-131).

Investigar desde el nosotros, accediendo a una verdad desde una transformación del yo, ha hecho que Huerting se convirtiera en un espacio de transformación y creación social (De Vita, 2012) que nos ha aportado claves en cuanto al acto de investigar y al acto de formar docentes en las aulas académicas.

\section{Hilos de Sentido de la Experiencia}

Habitar en comunidad la práctica investigativa de nuestros trabajos doctorales, ha requerido de una constelación de encuentros y condiciones (Noddings, 1987; Quiles-Fernández, 2016), gracias a la que, desde el presente, podemos recontar (para revivir) nuestras trayectorias en Huerting como experiencias transformativas. Hablamos de una constelación compuesta por los hilos de sentido que se han desprendido de la experiencia de investigar y escribir cada una de nuestras tesis doctorales; hilos que han trazado un recorrido propio, pero que han llevado a un despertar común en el pensamiento pedagógico y que, en esta mirada hacia atrás, hemos podido contemplar cómo han hecho trama hasta formar un tejido de saberes (Contreras \& Pérez de Lara, 2010). 


\section{La Experiencia de Roxana Hormazábal Fajardo: otro Modo de Vivir lo Académico y Nombrar la Experiencia}

Aprender a nombrar la experiencia demanda modos de pensar en plural, ya no solo apegarse a esa perspectiva individualista que se ha instalado desde la escuela racionalista-técnica para explicar el mundo. La academia y la formación universitaria han insistido en visibilizar un modo de construir y nombrar el conocimiento que ha originado un discurso y un método dominante para representar lo que se produce y acontece en él. En mi trayectoria escolar y universitaria observo esa prominencia del conocimiento científico racional, abstracto, neutral y objetivo que, sin duda, obstaculizó mi encuentro en ese nuevo espacio de pensamiento que cultivaba los saberes de manera abierta y natural.

Huerting ha sido una experiencia de formación vivida con un pie adentro y otro afuera de los muros institucionales, que significó una transformación en ese modo de estar y hacer academia, a partir de lo que también traemos a ella y no solo de lo que ella nos da. Puede ser cuestión de matices, pero esto ha dejado una resonancia significativa desde que fuimos tomando consciencia del espacio que íbamos creando colaborativamente.

Zambrano (2011) diferencia lo que conocimiento y saber nos aportan en nuestro crecimiento y camino de vida en torno al pensar. El conocimiento tiene un carácter puramente intelectual, es algo a lo que se accede cuando se tiene la disponibilidad de adquirirlo; el saber, en cambio, es una cualidad inherente a la vida misma del ser humano, pues se trata de una "experiencia ancestral o experiencia sedimentada en el curso de una vida" (p.147), lo que implica que el saber puede ser una herencia que hombres y mujeres nos vamos legando según vivimos la vida. Esa frontera, a veces sutil y otras confusa, que se marcaba entre conocimiento y saber, nos hacen distintos cuando nos situamos en uno u otro lugar, porque son diferentes modos de comprender el mundo que afectan especialmente cuando hablamos de investigación educativa, donde la voz del otro o de la otra siempre es importante. En Huerting hacíamos precisamente lo opuesto, dejábamos fluir las voces (y los silencios) de cada uno, acompañándonos de la experiencia que íbamos entretejiendo en ese espacio y en las investigaciones que estábamos desarrollando. 
Estar en relación y sostener un pensamiento a partir del encuentro con los otros nos desplaza de esa individualidad que impone habitualmente la academia, sobre todo si observamos los programas de formación doctoral en el Espacio Europeo. Estar en una relación pensante con los otros y con el propio saber (Mortari, 2002), es poner en juego la interioridad con la exterioridad para iniciar el reconocimiento de la construcción de un yo a partir de un nosotros (De Montaigne, 2010); por tanto, esto demanda abrir el horizonte del lenguaje a otros modos de dejar salir la palabra dicha y escrita, a ser conscientes del lugar que adquiere el cuerpo en esa búsqueda de comunicación. Estar en relación también significó desencuentros, perplejidad y silencios, por lo que es importante entenderlo como un camino no exento de obstáculos, pues los procesos que implican un despertar o un redescubrimiento incluyen esas detenciones como parte sustancial de lo que significa aprender.

En este andar, ninguno cuestionó el modo en que empezamos a trabajar, probablemente, porque la mayoría éramos docentes en algún nivel escolar o universitario, y nuestra labor la hacemos siempre en relación. Estar en relación era algo tan natural y cotidiano que, quizás por lo mismo, no lo habíamos problematizado como una metodología para cultivar el pensamiento. Sin embargo, contenía un saber hacer que emergía con cierta fluidez, probablemente impulsado por el hecho de no predeterminar una estructura sobre cómo y qué debía hacer cada uno en ese espacio.

Algo fundamental que sostuvo este proceso de transformación, fue darle lugar a la pregunta por el sentido pedagógico (Contreras, 2010). Ha sido clave dejar que las preguntas habiten en cada quién (Gadamer, 1977; Van Manen, 2003) y no solo sean consideradas un inicio para llegar a un final, es decir, reproducir la correspondencia pregunta-respuesta de la ciencia tradicional. Lo central en esto era que mantener las preguntas vivas permanentemente, nos permitía abrir un diálogo que tuviera sentido; que posibilitaba el entretejido (en contraposición a la fragmentación del conocimiento que prima en cualquier proceso de formación) colectivo e individual de lo que se conoce con lo que se sabe, de lo que se piensa con lo que se vive, de lo académico con lo escolar, de la experiencia con el saber.

La pregunta viva, el preguntarnos una y otra vez, nos daba la oportunidad de abrirnos a la escucha no solo del otro, sino de lo que la experiencia nos estaba diciendo para repensarnos, reconstruirnos, transformarnos y prepararnos para nuestro nacimiento como 
investigadoras/es que buscamos sensibilizarnos, comprender, visibilizar y repensar lo pedagógico apegándonos al sentido propio de la vida que se vive en las escuelas o en la formación del profesorado. "Nacimiento es siempre separación", dice Zambrano (2012, p. 53), en este sentido, hablamos de nacer para desplazarnos de aquel modo de entender la educación como proceso fragmentado; nacer para ir hacia aquello que nos hace sentido; nacer para tomar distancia de ese modo más visibilizado de hacer academia (sin desconocer que todo eso otro ha sido parte del camino necesario), para poder crear un pensamiento que viene dado por la experiencia de estar en una relación consciente con el mundo.

\section{La Experiencia de Emma Quiles-Fernández: Conversar Partiendo de sí, en Presencia y en el Presente}

Cuando mis compañeros comparten sus textos en Huerting, tengo la necesidad de dejarme guiar por lo que están poniendo en juego con su escritura, y de preguntarme acerca de lo que me suscitan y dicen sus palabras (Diario reflexivo, 2015).

Ese doble ejercicio, el de dejarme guiar y preguntarme, desvela algo que va más allá de la escritura. Si bien es cierto que lo que nos ponía en relación eran nuestros textos, lo que permitía sostener dicha relación no eran las palabras, sino la escucha. Una escucha auténtica que en ocasiones ha necesitado de dilatados silencios antes de poder nombrar o dar respuesta a las cuestiones que se ponían en juego. Una escucha que permitía comprender algo más de nuestras investigaciones, así como de aquellos contextos educativos y sociales de los que formaban parte. Y una escucha que siempre se presentaba en relación.

Leer en voz alta, una práctica poco común en la academia hacía que nos dejáramos llevar no sólo por el sentido de lo que se estaba abriendo, sino por la voz lectora. Se trataba de escuchar la voz que daba luz a la palabra para poder así adentrarnos en un determinado paisaje educativo, sintiendo la sonoridad de lo que cada uno deseaba compartir aquel día. En aquella atmósfera, había quien cerraba los ojos, dejándose llevar por la narración, y quien apoyaba la espalda en la silla, donde los hombros se percibían relajados. Yo no podía soltar el bolígrafo. Sostenerlo era como estar 
escribiendo en el aire, simbólicamente, aquello que iba movilizándose en mi cuerpo.

El momento de la lectura era un momento mágico: todo permanecía en silencio y la humildad aparecía para honrar esa dedicación y cuidado con el que cada uno había elaborado su escritura. El acto de compartir nos colocaba en una posición de vulnerabilidad, porque exponíamos aquello que habíamos ido hilando, así como aquello que todavía no sabíamos, que se nos escapaba, que iba más allá de nosotros y que ni siquiera a veces éramos capaces de poder nombrar completamente. Recuerdo cómo, por momentos, la vulnerabilidad de los otros me volvía todavía más vulnerable, favoreciendo el ejercicio de repensarme una y otra vez, de recuperar experiencias pasadas que me servían de espejo para el presente, siendo consciente de que "I am... what I am not yet"5 (Greene, 1995).

Cada narración compartida hacía que los saberes encarnados salieran a la luz en forma de historias personales y profesionales, de experiencias que iban creando un tejido semi invisible similar al de una tela de araña. Era complejo tomar notas de lo que ocurría en los encuentros porque todo se iba conectando con tanta fluidez que, imbuidos, avanzábamos hacia delante y hacia atrás en espiral. Los escritos se mezclaban con nuestras investigaciones, con autores que nos ayudaban a pensar, con historias de vida que cada uno traía al espacio relacional, y con presiones académicas que situaban el valor de lo educativo en lo cuantificable. Movernos en ese entramado no era sencillo, sobre todo porque cada una conectaba diversamente. Sin embargo, el tejido se sostenía porque nos reconocíamos en las preguntas y en las inquietudes de los compañeros. Y la curiosidad y deseo por querer saber y comprender acrecentaba el placer de estar y aprender juntos. Nos gustaba "estar dentro del simbólico que estábamos construyendo" (Piussi, 2004, p.66).

En ese placer por estar íbamos co-componiendo historias que nos permitían volver a pensar la práctica pedagógica, no con la intención de innovarla o tecnificarla, sino a fin de mirarla, nombrarla, y experimentarla como nueva. Cuando me retiraba de la conversación para dar más espacio a otras voces, mi corazón latía con fuerza. Era ahí donde percibía con claridad la trascendencia de salir hacia el exterior, al encuentro del otro, no para descubrir su secreto, sino para ahondar en el propio (Quiles-Fernández, 2016). Sentía que estábamos pensando en conversación, haciendo que la escritura primero habitara en nuestros cuerpos para luego poder plasmarla 
en el papel. Y, mientras cultivábamos aquel espacio formativo, la experiencia de Huerting habitaba en nosotros.

Cada voz me ayuda a pensar algo (...) imagino qué me diría cada uno y, en ese escribir a solas, me pregunto con sus voces para abrir más el texto (Diario reflexivo, 2016).

Imaginarme acompañada me ayudaba a visibilizar el modo en que cada uno había transformado algo en mí. Tenerlos presentes en mi investigación posibilitaba una gestación de las condiciones adecuadas que no ahogaba la alteridad, sino que le daba un lugar en el que la relación educativa era provechosa y fértil (Montoya Ramos, 2008). Esas condiciones pasaban por un cuidado del lenguaje y de la escucha, llegando a la creación de un espacio íntimo y de confianza. Habitar ese espacio ligado a la experiencia tenía que ver con la afección. Un afecto que traía consigo la irrupción de lo inesperado sobre lo esperado, del choque sobre la quietud, de la existencia del otro a la presencia de uno. En este sentido, Nancy (2007) sostiene que "el contacto -la contigüidad, la fricción, el encuentro y la colisión- es la modalidad fundamental del afecto" (p. 51).

Viajar de nuevo a la sala donde nos reuníamos, y recordar los modos en los que me dejé afectar por mis compañeros, me hace pensar en cómo las comunidades de pensamiento hacen que nos vivamos en algo más grande que uno mismo. Y es que "our communities do more than guide us -they constitute us (...) they give us a language, a culture, an inheritance, a home (Lindemann, 1995, p.28) ${ }^{6}$.

Huerting creó para mí la posibilidad de pensarme en una mediación amorosa en la que he podido reconocerme y hacerme reconocible, es decir, en la que he podido ser y vivirme en libertad (Muraro, 2007).

\section{La Experiencia de Julio Hizmeri: el Cuidado del Lenguaje, la Escucha y las Resonancias}

"E imaginar un lenguaje significa imaginar una forma de vida" (Wittgenstein, 1988, p.31)

En esta cita, Wittgenstein nos ayuda a repensar el lenguaje como un modo de vida. Y, quizás, un modo de referir a la experiencia vivida en 
Huerting sea pensando en los lenguajes o formas de vida que poníamos (o no) en juego.

Cuando llegué a Huerting, el grupo existía desde antes. Y como suele ocurrir con quien arriba a una comunidad desde otro lugar, me sentí desintonizado con los modos de decir, pensar, escuchar que se ponían en juego. Recuerdo un comienzo silencioso y no por falta de palabras afables que invitaban a la conversación. Más bien vivía ese silencio no como un mutismo baldío, en el que las palabras de otras u otros se pierden sin dejar eco, sino como un silencio abierto a la escucha, ocupado en sintonizar con lo que se decía. Tenía la sensación de que las palabras de las otras y otros fluían en una conversación dentro de un horizonte de sentido común hacia el que no me era fácil adherir con mi lenguaje de entonces. Era como si a las palabras escuchadas no podía responder con mi lenguaje y ante el sentimiento de extrañeidad, sentía la necesidad de callar (Hizmeri, 2016).

Cuando ingresas a una comunidad hay que atravesar ante todo las fronteras del lenguaje. "La cuestión de la lengua es una cuestión importante (...) en ella se juega lo simbólico, la existencia simbólica de los sujetos y el sentido del mundo" (Piussi, 2001, p.150). En Huerting, al mismo tiempo que percibía la vitalidad de las palabras de otras y otros, sentía la falta de las mías. Mucho de lo que se decía pasaba por la experiencia, la subjetividad, la memoria, el cuerpo a través de palabras bien cuidadas que daban cuenta tanto de la atención sobre sí como sobre lo educativo, mientras yo debía aún dejar atrás un lenguaje impersonal y neutro que acusaba el desencuentro conmigo mismo, revelando cierta indiferencia entre ser y palabra.

Así, no sería raro que mis primeras palabras no fueran para hablar de una experiencia -que es siempre personal- sino para comentar algo de epistemología, a modo de sobrevuelo. Y si bien lo dicho tenía sentido, parecía cuerdo o sensato, comenzaba a sentir el deseo de despertar de nuevo a otro lenguaje y de volver a enunciar palabras cuya sonoridad me devolvieran la sensibilidad del oído, de la boca; o, mejor, mi relación con lo vivido. Experimenté la necesidad de emprender un camino diferente hacia un sentido que aunara el sentido sensato de lo que se piensa con el sentido sensible de lo que se expresa, pues "tal vez sea preciso que el sentido no se conforme con tener sentido (o ser logos), sino que además resuene” (Nancy, 2007, p.16). 
Era como si necesitara comenzar a hablar de nuevo, volver a nacer a un lenguaje vinculado a la experiencia y lanzarme a la búsqueda de la correspondencia entre sí, el lenguaje y las cosas dichas (Muraro, 2007). Así, cuando estaba habituado al lenguaje de la academia, comencé a sentirme extraño, deshabitando el lenguaje. Y si bien el lenguaje es la morada del ser (Heidegger, 2000), no es lo mismo morar en uno o en otro lenguaje.

En el caso de Huerting, se trataba de un lenguaje vinculado a lo vivido, al tiempo, a la memoria: "Lo que tú dices me recuerda a la maestra a la que estoy visitando...", "Con tu escrito he recordado mi infancia en la escuela...", etc. Tiempo y memoria, eran dos formas a través de las cuales se ponían en juego relatos de experiencia que hacían sentir que la verdad de lo que pensamos se mide más por las resonancias que por la sensatez de lo dicho (Hizmeri, 2016). Entendería que para estar allí no era útil el lenguaje académico acostumbrado. Y, aunque faltarán las palabras, una experiencia ineludible ha sido empezar tirando aquellas que sobraban; aquel lenguaje deshabitado que buscaba abrazar conceptualmente la vida para ahogarla en una explicación racional, dogmática. Después de todo, "si el pensamiento no barre la casa por dentro, no es pensar” (Zambrano, 1989, p. 82).

Así, más allá de la ciencia tradicional y sus lenguajes académicos, en Huerting se trataba de ensanchar el sentido mediante un lenguaje sensible a la experiencia, donde la escucha comenzó a abrir un canal que mi lenguaje aún tenían cerrado. Y si 'la escucha es siempre personal. 'Qué me dicen' no es solo, ni es exactamente lo mismo que 'qué dicen"' (Contreras, 2013, p.65), la resonancia ('el qué me dicen') se dio antes en la escucha que, en mi palabra, dando lugar a una verdad por encuentro de experiencias y sentido (sensato, sintiente): verdades por resonancia.

Por ello, pienso que han sido las resonancias las que han guiado nuestras medidas de la verdad o el sentido de nuestras búsquedas. Una verdad que llega tan lejos como el sentido que nos hace aquello que nos dicen o que nos decimos; tan lejos como nos resuene. Así, a distancia de la objetividad $\mathrm{y}$ verdad académicas, hemos experimentado el sentido formador $\mathrm{y}$ transformador de una verdad relacional que necesita, para crecer, de otras y otros. Y con la lengua del tiempo, del cuerpo y de la memoria hemos abierto el espacio a las resonancias y al sentido para intentar hacer visible y pensable las cuestiones educativas que nos movían como investigadores nacientes, quienes, junto a la presencia y guía de José Contreras, fuimos dando forma a ese modo de vida que nos fue formando, transformando. 


\section{Reflexiones Finales}

Hoy, donde cada uno de nosotros se encuentra en un contexto diferente y tratando de dar respuesta a un conjunto de necesidades universitarias diversas, descubrimos que mostrar la práctica pedagógica de una comunidad de pensamiento no deja de ser una cuestión compleja, sobre todo cuando lo vinculamos a nuestro desarrollo profesional en la vida académica. Y es que, mientras que la comunidad científica convencional coloca en el centro la búsqueda de aquello que desea poseer, de aquello que viene dado desde el exterior (el conocimiento), la comunidad de pensamiento solo es posible al tomar consciencia de nuestra condición de seres humanos, sujetos en búsqueda de sí que necesitan de un reconocimiento. Un reconocimiento del otro que se da a través de una relación auténtica. En este sentido, ser comunidad de pensamiento es ser en relación tanto con los otros como con el mundo y con el saber, pero para ello hay que hacer el recorrido por uno mismo, por quien se es. En las tres narrativas que trenzan el presente documento, se muestra cómo la propia voz actúa de puente para poder acudir al encuentro de otras voces. Voces con la que entretejer saberes, historias y preguntas vivas que se convierten en modos posibles desde los que comprender y vivir el mundo educativo e investigativo en plural. En otras palabras, la comunión no existe cuando soy solo yo conmigo mismo (Zambrano, 2011). Y una comunidad de pensamiento permite compartir experiencias, preguntas y tensiones que, en relación con otros, pueden ser transitadas y hacerse fructíferas (Clandinin, 2013).

En este sentido, la escritura de este artículo nos ha permitido no sólo recontar, sino revivir la experiencia formativa de Huerting y hacerla visible como un espacio educativo compartido, de crecimiento intelectual y pedagógico, que durante un tiempo cuidamos y disfrutamos. Al traer todo esto al presente, hemos podido visibilizar que nuestra experiencia investigativa no ha podido darse sin la intervención de un método (Zambrano, 2011) que abrió caminos tanto como orientó recorridos, y en virtud del cual cobran sentido las tres voces individuales. Experiencias individuales que, si bien dan cuenta de pasajes formativos singulares en torno la escritura de la tesis (y cuyos motivos recurrentes refieren a otro modo de vivir lo académico, la necesidad de partir de sí o de la experiencia, 
el cuidado del lenguaje o la manera de nombrar la experiencia, la conversación y la escucha, las vivencias relatadas y las resonancias) han podido suscitarse sólo dentro un horizonte más amplio que, a modo de señales teóricas, guiaron nuestro andar singular dentro de un estar-encomún. A saber: la necesidad de situarnos en un terreno fértil, más allá de la razón tradicional y las modalidades investigativas más academicistas, para restituir el sentido que tiene la experiencia en la investigación educativa; la conciencia de que el saber educativo necesita mantenerse en una relación viva con la experiencia, dando lugar a un "saber encarnado" (Johnson, 2014), un "conocimiento práctico personal" (Clandinin, 1985), un "saber de la experiencia" (Contreras \& Pérez de Lara, 2010), que ha sostenido nuestros procesos investigativos; el experimentar la indagación narrativa como método propicio para dar cuenta de lo que nos pasaba y a partir de allí continuar tejiendo y ensanchando el sentido de nuestras búsquedas y nuestra formación como investigadores; $\mathrm{y}$, finalmente, el valor de la profundización de narrativa para suscitar, atender y pensar lo educativo al hilo de las historias que íbamos contando, viviendo, recontando y reviviendo.

Repensar cómo co-compusimos un espacio que acogía los procesos singulares y abrazaba los saberes que se iban cultivando grupalmente, nos coloca de nuevo frente a una cuestión significativa acerca de los espacios formativos para estudiantes de doctorado. Sabemos que uno de los núcleos de la tarea académica es dar relieve pedagógico a las prácticas investigativas; hacerlas visibles, pensables y, al tiempo, hacernos más conscientes de los caminos y tropiezos de lo educativo, de sus delicados procesos y los destellos de sentido que hallamos en ellos. En nuestro caso, este modo de cuidar el saber pedagógico que nace de la realización del trabajo de tesis doctoral lo vivimos en relación. El camino que Zambrano (2011) nos invita a repensar también queda vinculado con la pregunta acerca del modo en que hemos venido atendiendo a las necesidades reales y sentidas que hemos experimentado como investigadores. Una pregunta que nace y se desarrolla en la academia desde múltiples perspectivas: ¿de qué modo cultivamos espacios universitarios en los que compartir reflexiones pedagógicas acerca de los procesos de investigación personales? ¿cómo creamos espacios que posibiliten un encuentro entre el pensamiento y la vida educativa, sin que el primero solape al segundo, o sin que los vivamos como dos mundos separados? Porque hacernos investigadores y 
comprender la investigación educativa como un proceso de transformación personal no solo requiere de una epistemología, sino también de espacios en donde poder cuidar y cultivar esa forma experiencial de hacer investigación y hacerse investigador e investigadora.

\section{Agradecimientos}

Queremos agradecer a José Contreras, por invitarnos a Huerting y ayudarnos a repensar el sentido del quehacer educativo; y a Susana Orozco-Martínez e Ilich Silva-Peña por la dedicación y respeto con el que han realizado aportaciones a este escrito.

\section{Notas}

${ }^{1}$ Nos consideramos “investigadores nacientes” (Hormazábal Fajardo, 2016) porque sentimos que durante los años de formación doctoral estuvimos naciendo a cada momento, partiendo de una experiencia propia y encarnada que ha posibilitado habitar la comunidad de pensamiento en presente y en presencia, promoviendo una particular relación pensante con el saber pedagógico puesto en juego en nuestros encuentros.

${ }^{2}$ Tal y como Huber et al. (2014) sostienen, el acto de indagar narrativamente nos permitió poder vivir, contar, volver a contar y revivir nuestros relatos de experiencia vinculados al proceso singularizado de nuestras tesis doctorales. En este sentido, "el potencial extraordinario de vivir, contar, volver a contar y revivir dichos relatos" (p. 33) nos lleva a considerar a la indagación narrativa como una pedagogía en sí. Además, siguiendo las aportaciones de Okri (1997) "we live by stories, we also live in them. One way or another we are living the stories planted in us early or along the way, or we are also living the stories we planted ... in ourselves" (p. 46).

${ }^{3}$ En Notas para un método, María Zambrano (1989) defiende un método como camino que no precede a la experiencia como un programa preestablecido, sino que emerge durante la experiencia.

4 Al nombre escogido hay que añadir el significado que otorga la expresión 'ing' de la lengua inglesa. El gerundio da cuenta de lo que estaba en movimiento en nuestra comunidad de pensamiento: continuar leyendo, conversando, escuchando, pensando, escribiendo, etc.

5 "Yo soy aquello que todavía, y de momento, no he llegado a ser" (Traducción propia de los autores del artículo).

6 "Nuestras comunidades hacen algo más que guiarnos -ellas nos constituyen (...) ellas nos ofrecen un lenguaje, una cultura, una herencia, un hogar" (Traducción propia de los autores del artículo). 


\section{References}

Arnaus, R. (2013). La relación como práctica política en la formación inicial de educadoras y educadores sociales. Revista Interuniversitaria de Formación del Profesorado, 78, 71-88. Recuperado de http://www.redalyc.org/html/274/27430309004/ Bárcena, F., \& Mèlich, J. C. (2014). La educación como acontecimiento ético. Natalidad, narración y hospitalidad. Buenos Aires: Miño y Dávila.

Behar, R. (1996). The vulnerable observer: Anthropology that breaks the heart. Boston, MA: Beacon Press.

Clandinin, D. J., \& Huber, J. (2012). Narrative inquiry. En B. McGaw, E. Baker \& P. P. Peterson (Eds.), International Encyclopedia of Education (pp. 436-441). New York: Elsevier.

Clandinin, D. J. (1985). Personal practical knowledge: A study of teachers' classroom images. Curriculum Inquiry, 15(4), 361-385.

doi:10.2307/1179683

Clandinin, D. J. (2013). Engaging in narrative inquiry. New York: Routledge.

Connelly. M., \& Clandinin, D. J. (1995). Relatos de experiencia e investigación narrativa. En J. Larrosa, R. Arnaus, V. Ferrer, N. Pérez de Lara, F.M. Connelly, D.J. Clandinin \& M. Greene, Déjame que te cuente. Ensayos sobre narrativa y educación (pp. 11-59). Barcelona: Laertes.

Contreras, J. (2010). Pedagogía de la experiencia y la experiencia de la pedagogía. En J. Contreras \& N. Pérez de Lara (Coord.) Investigar la experiencia educativa (pp. 241-269). Madrid: Morata.

Contreras, J. (2013). Ponerse a la escucha. Cuadernos de Pedagogía, 430, 63-65. Recuperado de http://www.redalyc.org/html/1531/153114254013/

Contreras, J. (2014). El óvulo de Huerting. Documento interno.

Contreras, J., \& Pérez de Lara, N. (2010). Investigar la experiencia educativa. Madrid: Morata.

Contreras, J. \& Quiles-Fernández, E. (2017). Vivir y profundizar experiencias de enseñanza desde una perspectiva narrativa. En Contreras, J. (coord). Enseñar tejiendo relaciones. Madrid: Morata. De Montaigne, (2010). Ensayos completos. Madrid: Cátedra. 
262 Quiles-Fernández et al. - Formarse en la Investigación Educativa

De Vita, A. (2012). La creación social. Relaciones y contextos para educar. Barcelona: Laertes.

Gadamer, H. G. (1977). Verdad y método. Fundamentos de una hermenéutica filosófica. Salamanca: Sígueme.

Gadamer, H. G. (2000). Educar es educarse. Barcelona: Paidós.

Greene, M. (1995). Releasing the imagination. Essays on education, the arts, and social change. San Francisco: Jossey-Bass.

Heidegger, M. (1978). ¿Qué es la filosofía? Madrid: Narcea.

Heidegger, M. (2000). Carta sobre el humanismo. Madrid: Alianza Editorial.

Hizmeri, J. (2016). Otro modo de estar en la relación educativa. La investigación del movimiento de autorreforma italiana de la escuela como una experiencia de transformación personal (Tesis doctoral). Universidad de Barcelona, España). Recuperado de: https://tdx.cat/handle/10803/387818

Hormazábal Fajardo, R. (2016). La historia que nos nace. Indagación narrativa con docentes en la experiencia de enseñar Historia (Tesis doctoral). Universidad de Barcelona, España. Recuperado de: https://tdx.cat/handle/10803/393950

Huber, J., Caine, V., Huber, M., \& Steeves, P. (2014). La indagación narrativa como pedagogía en la educación: el potencial extraordinario de vivir, contar, volver a contar y revivir relatos de experiencias. Revista de Educación, 7, 33-74. Recuperado de https://fh.mdp.edu.ar/revistas/index.php/r_educ/article/view/981

Johnson, M. (2014). Embodied knowledge. Curriculum Inquiry, 19(4), 361377. doi:10.1080/03626784.1989.11075338

Larrosa, J. (2003). Entre las lenguas: lenguaje y educación después de Babel. Barcelona: Laertes.

Lindemann, H. N. (1995). Resistance and insubordination. Hypatia, 10, 23 40. Retrieved from https://philpapers.org/rec/NELRAI

Merleau-Ponty, (2013). El ojo y el espíritu. Madrid: Trotta

Montoya Ramos, M. M. (2008). Enseñar: una experiencia amorosa. Madrid: Sabina Editorial.

Mortari, (2002). Tras las huellas de un saber. En Diótima El perfume de la maestra: en los laboratorios de la vida cotidiana (pp. 153-162). Barcelona: Icaria. 
Muraro, L. (2007). El pensamiento de la experiencia. Duoda. Estudis de la diferència sexual, 33, 41-46. Recuperado de https://dialnet.unirioja.es/servlet/articulo?codigo $=2486771$

Nancy, J. L. (2000). La comunidad inoperante. Santiago de Chile: Ediciones LOM.

Nancy, J. L. (2007). A la escucha. Buenos Aires: Amorrortu Editores.

Noddings, N. (1987). Caring. A feminine approach to ethics and moral education. USA: University of California Press.

Okri, B. (1997). A way of being free. London: Head of Zeus.

Piussi, A. M. (2000). Partir de sí: necesidad y deseo. Duoda. Estudis de la diferencia sexual, 19, 31-46. Recuperado de https://dialnet.unirioja.es/servlet/articulo?codigo=2652428

Piussi, A. M. (2001). Dar clase: el corte de la diferencia sexual. En N. Blanco (Coord.) Educar en femenino y en masculino (pp. 145-163). Madrid: Akal.

Piussi, A. M. (2004). Cómo establecer relación con los compañeros. En M. M. Montoya (Coord.), Recetas de relación: educar teniendo en cuenta a la madre (pp. 66-68). Madrid: Sofías.

Porres, A. (2012). Relaciones pedagógicas en torno a la cultura visual de los jóvenes. Barcelona: Octaedro.

Quiles-Fernández, E. (2016). Cuidar la relación: El sentido de la experiencia educativa. Una investigación narrativa (Tesis doctoral). Universidad de Barcelona, España. Recuperado de: https://tdx.cat/handle/10803/393960

Ricoeur, P. (2004). Tiempo y narración. México DF: Siglo XXI. Rubinstein-Avila, E., \& Maranzana, S. (2015). Dual Reflections on Teaching and Learning of Autoethnography: Preparing Doctoral Students Authentically for a Career in the Academy. Qualitative Research in Education, 4(3), 243-268. doi:10.17583/qre.2015.1328 Simons, M., \& Masschelein, J. (2006). Sobre el precio de la investigación pedagógica. En J. Masschelein \& M. Simons. Mensajes e-ducativos desde tierra de nadie (pp. 129-145). Barcelona: Homo Sapiens.

Van Manen, M. (2003). Investigación educativa y experiencia vivida. Barcelona: Idea Books.

Wittgenstein, L. (1988). Investigaciones filosóficas. Barcelona: Crítica. Zamboni, C. (2009) Pensare in presenza. Conversazioni, luoghi, improvvisazioni. Nápoles: Liguori. 
Zambrano, M. (1989). Delirio y destino. Madrid: Mondadori.

Zambrano, M. (2004). La razón en la sombra: antología crítica. Madrid:

Siruela.

Zambrano, M. (2011). Notas de un método. Madrid: Tecnos

Zambrano, M. (2012). Hacia un saber sobre el alma. Madrid: Alianza

Literaria.

Emma Quiles-Fernández is the Horowitz Teacher Education Research Scholar (2016-2018) and a teacher educator at the Centre for Research for Teacher Education and Development at the University of Alberta, Canada. ORCID, id: 0000-0002-9401-4387

Julio Hizmeri Fernández is Academic of the Department of Didactics, Faculty of Education at Universidad Católica de la Santísima Concepción, Chile. ORCID, id: 0000-0003-3729-376X

Roxana Hormazábal Fajardo is Associate Professor in the School of Primary Education at Faculty of Pedagogy, Universidad Academia de Humanismo Cristiano, Chile.

Contact Address: Emma Quiles-Fernández, Centre for Research for Teacher Education and Development, University of Alberta, 633 Education Building South, Edmonton, Alberta, T6G 2G5, Canadá. Email: emmaqe@gmail.com 